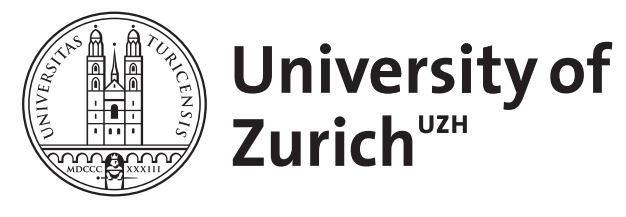

\title{
Technical errors in MR arthrography
}

\author{
Hodler, J
}

\begin{abstract}
This article discusses potential technical problems of MR arthrography. It starts with contraindications, followed by problems relating to injection technique, contrast material and MR imaging technique. For some of the aspects discussed, there is only little published evidence. Therefore, the article is based on the personal experience of the author and on local standards of procedures. Such standards, as well as medico-legal considerations, may vary from country to country. Contraindications for MR arthrography include pre-existing infection, reflex sympathetic dystrophy and possibly bleeding disorders, avascular necrosis and known allergy to contrast media. Errors in injection technique may lead to extra-articular collection of contrast agent or to contrast agent leaking from the joint space, which may cause diagnostic difficulties. Incorrect concentrations of contrast material influence image quality and may also lead to non-diagnostic examinations. Errors relating to MR imaging include delays between injection and imaging and inadequate choice of sequences. Potential solutions to the various possible errors are presented.
\end{abstract}

DOI: https://doi.org/10.1007/s00256-007-0329-z

Posted at the Zurich Open Repository and Archive, University of Zurich ZORA URL: https://doi.org/10.5167/uzh-11380

Journal Article

Published Version

Originally published at:

Hodler, J (2008). Technical errors in MR arthrography. Skeletal Radiology, 37(1):9-18.

DOI: https://doi.org/10.1007/s00256-007-0329-z 


\title{
Technical errors in MR arthrography
}

\author{
Juerg Hodler
}

Received: 21 July 2006 /Revised: 16 April 2007 / Accepted: 18 April 2007 / Published online: 26 May 2007

(C) ISS 2007

\begin{abstract}
This article discusses potential technical problems of MR arthrography. It starts with contraindications, followed by problems relating to injection technique, contrast material and MR imaging technique. For some of the aspects discussed, there is only little published evidence. Therefore, the article is based on the personal experience of the author and on local standards of procedures. Such standards, as well as medico-legal considerations, may vary from country to country. Contraindications for MR arthrography include pre-existing infection, reflex sympathetic dystrophy and possibly bleeding disorders, avascular necrosis and known allergy to contrast media. Errors in injection technique may lead to extra-articular collection of contrast agent or to contrast agent leaking from the joint space, which may cause diagnostic difficulties. Incorrect concentrations of contrast material influence image quality and may also lead to nondiagnostic examinations. Errors relating to MR imaging include delays between injection and imaging and inadequate choice of sequences. Potential solutions to the various possible errors are presented.
\end{abstract}

Keywords Arthrography · Magnetic resonance imaging · Contraindications $\cdot$ Infection $\cdot$ Hypersensitivity . Contrast media

J. Hodler $(\bowtie)$

Radiology, Orthopaedic University Hospital of Balgrist,

Forchstrasse 340,

8008 Zurich, Switzerland

e-mail: juerg.hodler@balgrist.ch

\section{Introduction}

MR arthrography is commonly used for imaging of the shoulder, but it is also used for imaging of other joints, including the hip, the wrist, the elbow, the ankle and the knee [1-3]. This article deals specifically with the technical aspects of direct MR arthrography (with images obtained after intra-articular injection of contrast material). Indications for MR arthrography and interpretation errors are not the subject of this article, and neither is the indirect form of MR arthrography where imaging is performed after intravenous injection of contrast medium.

There are four sections, including contraindications as well as problems relating to injection technique, contrast media and MR examination technique. Potential solutions to these problems are discussed.

Because published evidence is scarce for some of the subjects discussed, this article is, in part, based on personal experience and on local standards of procedure. The reader should be aware that rules and regulations, as well as availability of contrast material, vary from country to country.

\section{Contraindications for MR arthrography}

Infection

MR arthrography should not be performed where there is suspected infection of the skin or soft tissue close to the needle path. The needle may carry infectious agents into a previously non-infected joint; for instance, into the glenohumeral joint if there is infection of the subdeltoid bursa. When septic arthritis is already present, the increased intraarticular pressure caused by the injected contrast material causes pain, and there is at least the theoretical possibility 
of haematogeneous propagation of infection. In addition, intra-articular contrast material does not add diagnostic information in septic arthritis. Early signs of synovitis may even be obscured in the presence of hyperintense intraarticular contrast material. In septic arthritis, the typically present joint fluid serves as a natural contrast material. Intravenous injection of contrast medium is useful in the presence of soft tissue and joint infections, because even subtle synovitis may be demonstrated.

Reflex sympathetic dystrophy

Reflex sympathetic dystrophy may be reactivated after joint injection. The Reflex Sympathetic Dystrophy Syndrome Association, in their guidelines for patients and hospitals (http://www.rsds.org, accessed on January 26, 2006) do not refer specifically to arthrography but recommend avoidance of all types of even minor injuries to the affected limb, including phlebotomy and cuff placement for blood pressure measurement. At our institution, the direct injection of contrast agent is avoided in reflex sympathetic dystrophy.

\section{Anticoagulation}

Occasionally, patients referred for MR arthrography have oral anticoagulant therapy. There is no general agreement regarding handling of oral anticoagulant therapy before interventions [4]. Even for gastrointestinal endoscopy with biopsy, discontinuation of anticoagulant therapy is not strictly indicated [4]. For the musculoskeletal system, little data have been published. Thumboo and O'Duffy [5] have evaluated 32 joint or soft tissue aspirations in patients undergoing warfarin sodium therapy. Four weeks after the procedure, no bleeding was found. The reduction of anticoagulation is not without risk and has to be related to the small risk of a joint injection. A very careful approach would be based on the policies of our institution's orthopaedic surgeons, who like to see an international normalized ratio (INR) value of approximately 1.5 or smaller for their interventions. Another approach includes careful discussion of indications for MR arthrography (which may be replaced by standard MR imaging or by indirect MR arthrography) and the reduction of the risk of bleeding by the use of thin needles and by having experienced radiologists to give the injection.

Avascular necrosis of bones adjacent to the injected joint

Based on our experience, arthrography is painful in avascular necrosis, presumably due to the increased intraarticular pressure caused by the injected contrast material. There are no data indicating that increased joint pressure or contrast material itself promotes avascular necrosis. Occa- sionally, avascular necrosis may occur after intra-articular injection of triamcinolone in children with childhood arthritis which cannot directly be compared to MR arthrography [6]. We rather avoid MR arthrography in avascular necrosis, because there is little additional information after the injection of intra-articular contrast medium. In one paper, better delineation of cartilage damage has been described [7]. However, because there is an increased amount of joint fluid in avascular necrosis serving as natural contrast material, we tend not to use intra-articular contrast agent in patients with avascular necrosis.

\section{Allergy to contrast media}

Allergic reactions to intra-articular contrast media is another subject of debate: Hasselbacher and Schumacher [8] found inflammation and eosinophilia of the synovial fluid following conventional arthrography of a gouty joint. They postulated that non-immune mechanisms were responsible for this finding. Newberg et al. sent questionnaires to 84 radiologists experienced in (conventional) arthrography; 57 respondents, representing more than 126,000 arthrographic procedures, found 61 cases of hives and rarely other potentially allergic reactions, including laryngeal edema. To our knowledge, there are no documented allergic reactions to intra-articular gadoliniumcontaining contrast media. Even if present, allergic reactions may rather originate from the injected local anaesthetics or the iodine-containing contrast agents used for demonstration of intra-articular needle position.

Even if allergic reactions to intra-articular gadolinium is not proven, they cannot be excluded. Manufacturers of gadolinium-containing intra-articular contrast media include a warning regarding allergic reactions. Therefore, some caution is recommended.

When a history of allergic reaction to contrast agent injections is reported, the gadolinium injection may have to be replaced by an injection of saline or Ringer's solution [9, 10], or standard MR imaging may have to be performed. If allergic reactions appear to relate to local anaesthetics rather than to contrast material, the arthrogram may be performed without local anaesthetics (using a thin needle and an experienced radiologist) or by changing the type of local anaesthetic.

Gadolinium-based MR contrast agents and nephrogenic systemic fibrosis

Nephrogenic systemic fibrosis (NSF) is a severe disease that has been associated with the intravenous injection of gadolinium-based contrast agent and renal insufficiency. Gadodiamide has most commonly been administered in patients with NSF. NSF is characterized by increased 


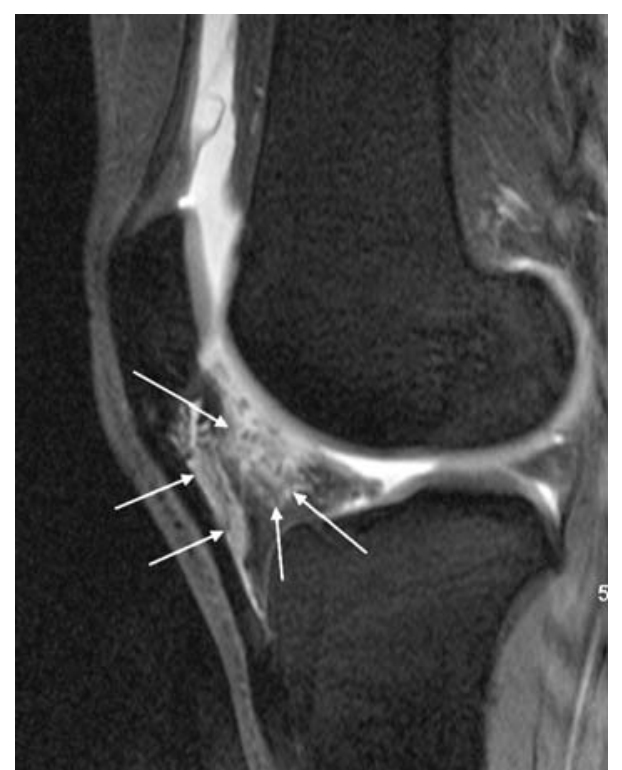

Fig. 1 MR arthrography of the knee, confounding effect of local anaesthetics. Sagittal, water-excitation, true fast imaging with steadystate precession (trueFISP) image of the knee, after injection of gadopentetate through an anterior infrapatellar route. Gadopentetate is hyperintense, as also are local anaesthetics injected into Hoffa's fat pad during needle advancement (arrows)

amounts of collagen, most commonly in the skin of the extremities but also involving other tissues (including the lung, skeletal muscle, the heart and others) [11]. To the best of our knowledge, no cases of NSF after intra-articular injection of gadolinium-based contrast media have been reported to date. Because the contrast material is diluted for MR arthrography, the amount of gadolinium potentially deposited in the skin is much smaller than after intravenous injection.

\section{Problems relating to injection technique}

\section{Pain during injection}

Local anaesthetics cause a burning sensation during the initial phase of injection. Therefore, rather small amounts of local anaesthetics should be injected, and the injection should be performed slowly. The effect of local anaesthetics starts after approximately half a minute. Adequate waiting time is required before the needle is further advanced. In general, pain experience during injection is not severe. It tends to be approximately $15-25$ on a visual analogue scale of $1-100$ [12]. Based on a patient questionnaire and personal experience, pain depends on the operators and their techniques, as well as on the patient. Needles touching bones cause pain. Therefore, bones close to or within the needle track have to be identified and avoided when one is planning the injection path. The coracoid is a typical example. Pain may be caused when a superior approach to the glenohumeral joint is chosen for injection. Close to bones, the injection volume of local anaesthetics may have to be increased.

Confounding MR appearance of extra-articular local anaesthetics

On short-tau inversion recovery (STIR) images, some gradient-echo as well as fat-suppressed proton-density and T2-weighted spin-echo images, local anesthetics may appear as a bright signal within soft tissue (Fig. 1). Rarely, this signal abnormality may mimic a pathological condition. Typically, such signal can be differentiated from disease because it is comparably subtle, relates to the needle tract and disappears on the T1-weighted sequences.

\section{Extra-articular injection of contrast agent}

What appears to be the joint space during fluoroscopy can sometimes not be reached by a needle oriented parallel to the X-ray because many joint spaces are curved, such as the distal radio-ulnar joint (Fig. 2) and the radiocarpal and ankle joints (Fig. 3). As an example, if the needle is aimed directly at the apparent ankle joint space, it will hit the distal anterior edge of the tibia. The solution to this problem is simple. The needle does not need to enter the joint space proper but can be placed anywhere within the synovial
Fig. 2 Injection of the distal radio-ulnar joint. a Fluoroscopic image; $\mathbf{b}$ corresponding axial, water-excitation, true fast imaging with steady-state precession (trueFISP) image. In a, the needle is not pointing at the apparent joint space but rather a few millimetres to the ulnar side. The axial image shows that passage of the needle into the joint would otherwise be obstructed by the dorsal radius, as shown in b (arrow)
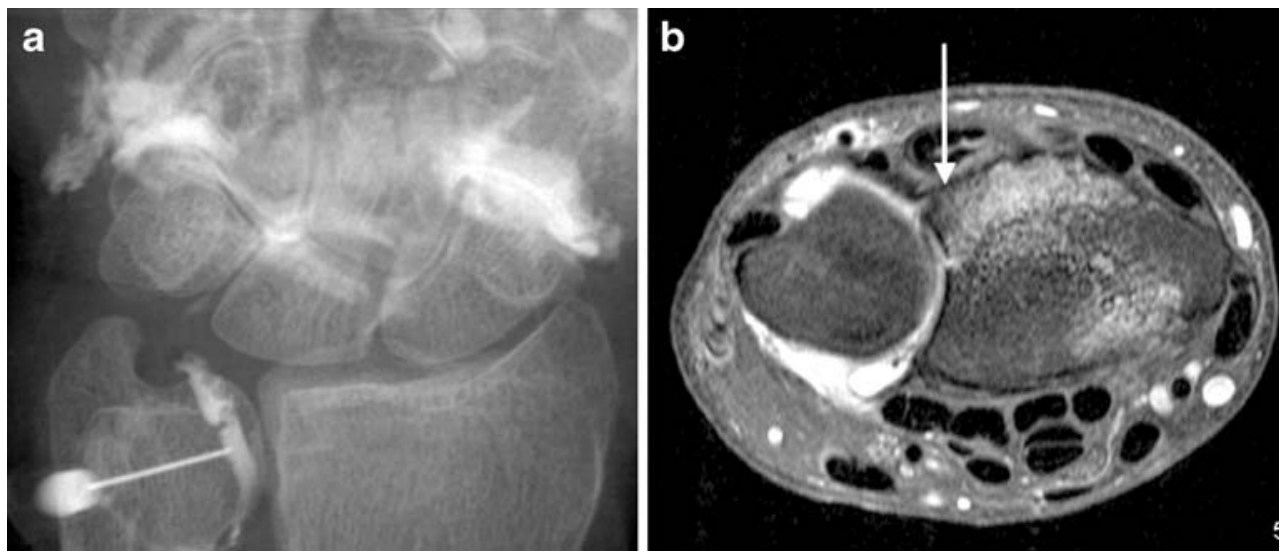


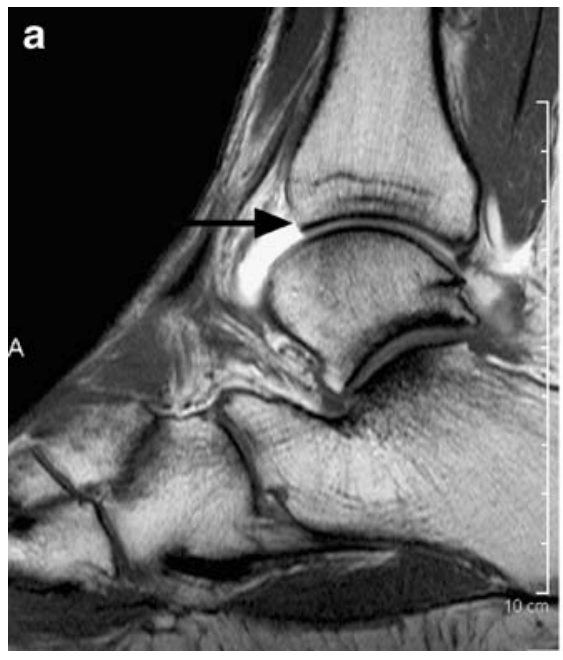

Fig. 3 Avoidance of the antero-inferior lip of the tibia during injection of the ankle joint. a Sagittal T1-weighted spin-echo image after intraarticular injection of contrast material; $\mathbf{b}$ injection technique for the antero-posterior (AP) position of the foot and $\mathbf{c}$ injection technique for lateral position of the foot. The arrow in a demonstrates that the needle would hit the anterior inferior rim of the tibia if aimed at the apparent

space. In the distal radio-ulnar joint, the needle can be placed a few millimeters ulnarly from the apparent joint space (Fig. 2). In the ankle joint the needle can be placed slightly inferiorly to the apparent joint space if the anteroposterior approach is employed. Another solution is to rotate the tube, or the patient, which will result in a lateral view of the ankle. This allows angulation of the needle, thus avoiding the prominent antero-inferior tip of the tibia (Fig. 3c). Knowledge of the anatomy of capsular

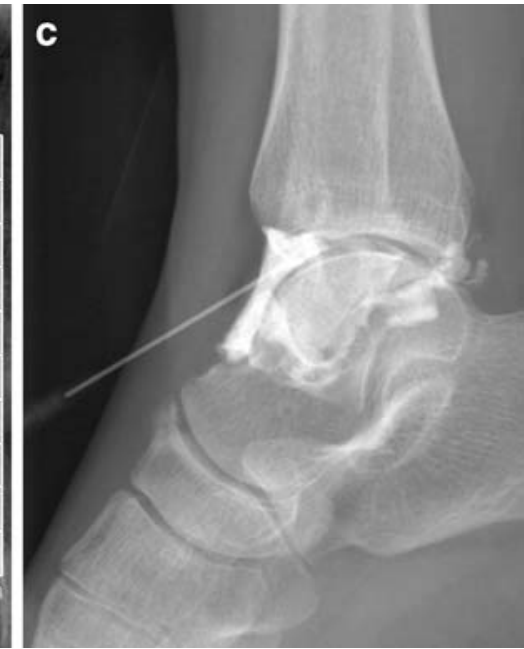

joint space during AP fluoroscopy; b demonstrates a solution to this problem (point the needle into the anterior recess, plantar to the apparent joint space); c demonstrates an alternative method, where the patient is lying on his side, and plantar-cranial needle angulation is being used in order to avoid the tibial rim

insertions is obviously important. As an example, in pronounced internal rotation of the humeral head, the humeral insertion of the capsule may be far medial and the needle tip may be located within the capsular insertion. Table 1 shows the anatomical structures potentially limiting access to joints.

The labrum of the glenohumeral and hip joints may also interfere with correct needle position. When the needle is aimed directly at the glenohumeral joint space, the needle

Table 1 Anatomical structures potentially limiting access to joints (DRUJ distal radio-ulnar joint)

\begin{tabular}{|c|c|c|}
\hline Joint & Problem & Solution \\
\hline \multirow[t]{4}{*}{ Glenohumeral } & Coracoid & Inject inferiorly, superiorly or laterally \\
\hline & Labrum & Do not try to reach the apparent joint space directly \\
\hline & Biceps tendon & Check fluoroscopic image and injection pressure \\
\hline & Internal rotation of humerus & $\begin{array}{l}\text { Choose neutral or internal rotation in order to move capsular insertion } \\
\text { laterally }\end{array}$ \\
\hline \multirow[t]{2}{*}{ Hip } & Labrum & $\begin{array}{l}\text { Identify anterior acetabular rim and choose a sufficient distance between } \\
\text { the rim and the needle }\end{array}$ \\
\hline & Orbicular zone & $\begin{array}{l}\text { Inject at superolateral femoral head [11] instead of the often described } \\
\text { injection site at the transition between femoral head and neck }\end{array}$ \\
\hline \multirow[t]{2}{*}{ Knee } & $\begin{array}{l}\text { Lateral parapatellar approach: leakage of contrast } \\
\text { material into pre-femoral fat }\end{array}$ & Choose more inferior injection site or tilt needle inferiorly \\
\hline & $\begin{array}{l}\text { Anterior infrapatellar approach: needle in soft } \\
\text { tissue of intercondylar groove }\end{array}$ & Aim needle at antero-inferior femoral cartilage (either medial or lateral) \\
\hline Ankle joint & Needle hits anterior distal rim of tibia & $\begin{array}{l}\text { Aim needle at anterior surface of talar dome, not at the apparent joint } \\
\text { space. Alternatively, use lateral fluoroscopy to control plantar-cranial } \\
\text { needle tilt }\end{array}$ \\
\hline DRUJ & $\begin{array}{l}\text { Needle hits anterior surface of radius when one is } \\
\text { aiming at apparent joint space }\end{array}$ & Aim at ulnar head \\
\hline $\begin{array}{l}\text { Radiocarpal } \\
\text { joint }\end{array}$ & $\begin{array}{l}\text { Needle hits dorsal distal rim of radius when } \\
\text { apparently being aimed at the radiocarpal joint } \\
\text { space }\end{array}$ & Aim at scapoid bone and tilt needle proximally after passing the radial rim \\
\hline
\end{tabular}


tip will hit the labrum (Fig. 4). In our experience this is painful, and contrast material may not be injected intraarticularly but rather into peri-articular tissue. In the hip, when the anterosuperior femoral head is used as target for the injection (as is performed at our institution), the needle should not be placed close to the femoral rim because it may be pushed into the labrum of the hip.

Another obstacle in the shoulder joint is the coracoid process, which may be quite long (Fig. 5). The coracoid can easily be avoided by a more superior, inferior or lateral injection site if the coracoid is correctly identified prior to the injection.

In the glenohumeral joint the needle may be located within the intra-articular part of the biceps tendon (Fig. 6). Such needle misplacement is quickly recognized on fluoroscopic images. Injection into the superior border of the subscapularis tendon is more common. It occurs when an anterosuperior approach to the glenohumeral joint is employed. The resulting hyperintensity may be misinterpreted as tendinopathy (Fig. 7). For this reason, the use of a posterior approach to the glenohumeral joint has been suggested [13]. When the anterior approach is preferred (as in our institution), contrast medium must be unequivocally flowing into the joint space during fluoroscopic control.

\section{Injection volume}

The typical injection volumes used at our institution are listed in Table 2. In the glenohumeral joint patients with large rotator cuff tears or patients with recurrent dislocation may require larger volumes in order to provide the desired joint distension. In the hip the use of more than $10 \mathrm{ml}$ has been suggested [14], in order to better demonstrate articular

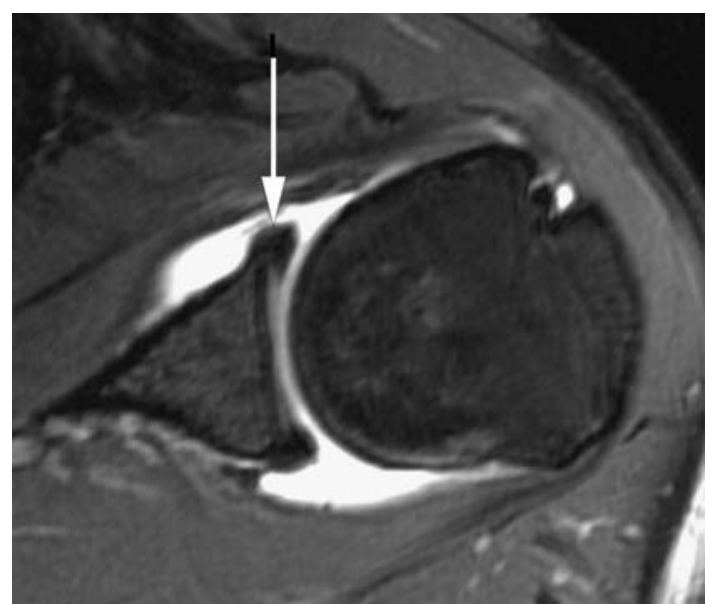

Fig. 4 Avoidance of the labrum of the glenohumeral joint. Axial, water-excitation, true fast imaging with steady-state precession (trueFISP) image of the left shoulder.The arrow demonstrates potential contact between needle and anterior labrum if the radiologists tries to reach the joint space as it appears under fluoroscopic guidance. For this reason, a more lateral needle position is desirable

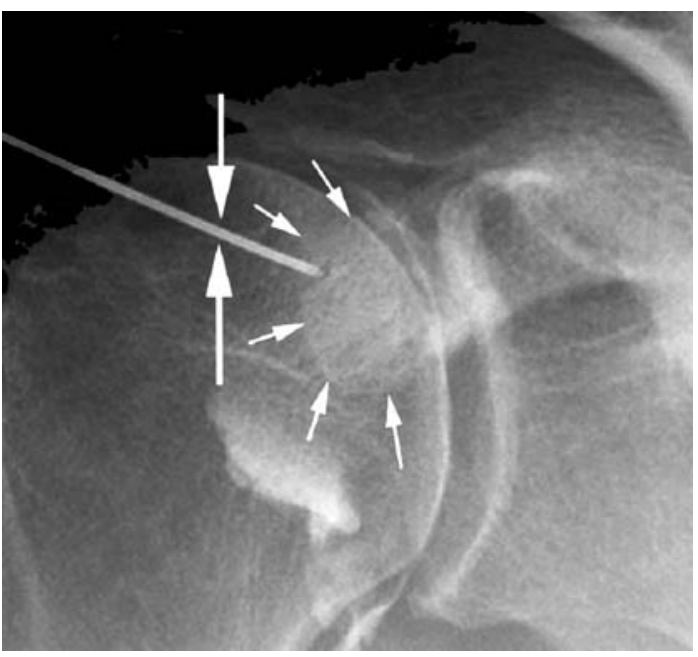

Fig. 5 Avoidance of a prominent coracoid process during glenohumeral joint injection. We prefer the anteriosuperior injection site, which, in this case, is obstructed by a prominent coracoid process (small arrows). The skin entry point was chosen more laterally than usual (two large arrows), and the needle was angled medially

cartilage. However, many patients complain of pain if the volume is too high. In the ankle the optimal volume depends on the presence or absence of communications that may normally be present with regard to the subtalar joint and with regard to tendon sheaths after trauma, such as the peroneal tendon. After trauma or surgery with scarring and in frozen shoulder, the indicated volumes need to be reduced. When injection volumes have been determined, the radiologists have to take into account the small amounts of local anaesthetics and iodine-containing contrast agents

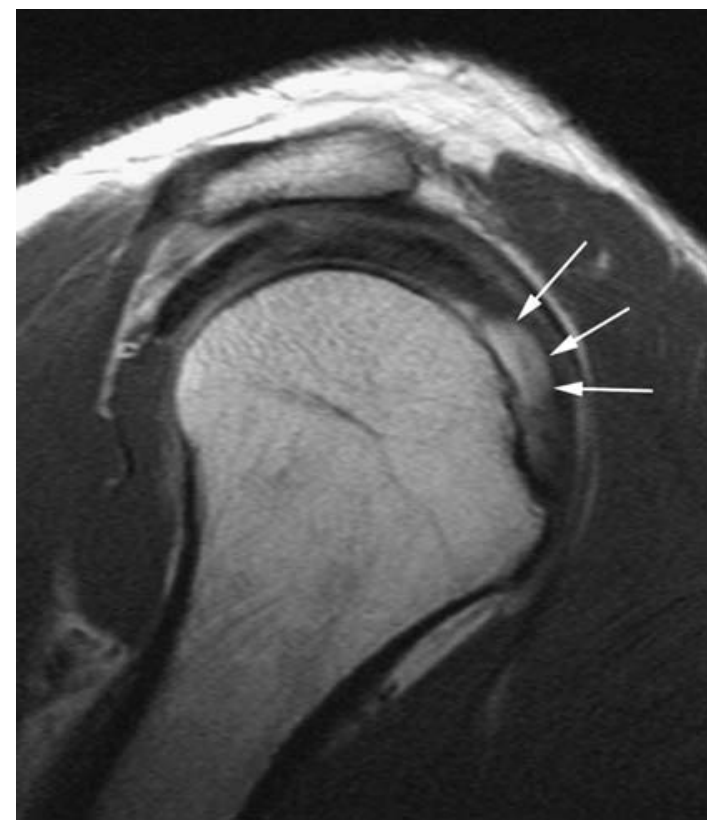

Fig. 6 Injection of contrast into biceps tendon; oblique angled, sagittal, T1-weighted image. Arrows point to the hyperintense biceps tendon. Rarely, contrast material may be injected partially into the tendon when the superior approach to the glenohumeral joint is used 


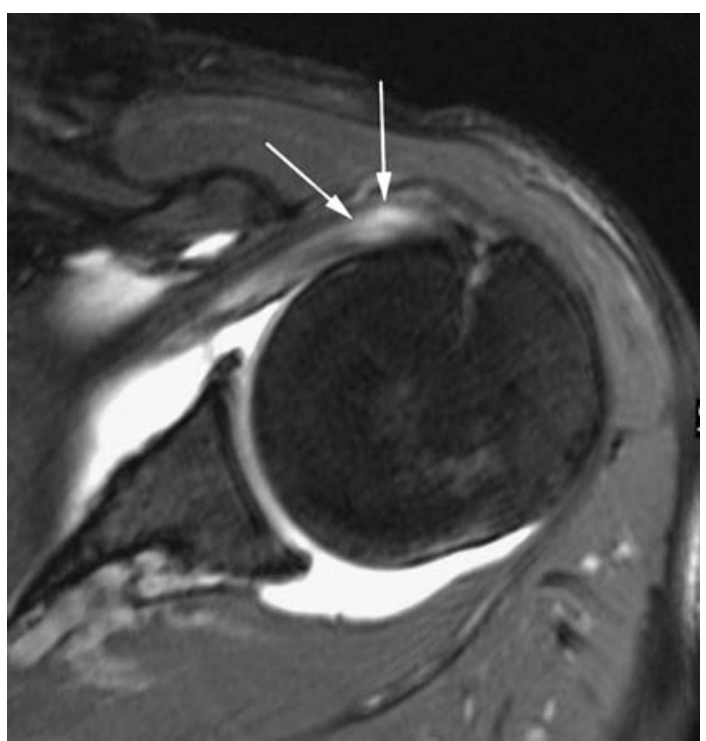

Fig. 7 Injection of contrast agent into the subscapularis tendon. Axial, water-excitation, true fast imaging with steady-state precession (trueFISP) image obtained after injection of gadolinium-containing contrast material. There is hyperintensity within the subscapularis tendon close to the humeral insertion (arrows). This occurs when the arm is internally rotated during injection

and pre-existing joint fluid. This is especially important in small joints, such as the distal radio-ulnar joint. Pre-existing joint fluid is most pronounced after trauma, in the presence of osteoarthritis or in large rotator cuff tears. We try to aspirate excessive fluid but do not insist on complete removal, which prolongs the procedure, may be painful and may introduce small air bubbles into the joint.

Insufficient volumes of contrast agent may result in too low concentrations of gadolinium and may not sufficiently distend the joint for the desired diagnosis. If volumes are too large, contrast material leaking from the joint may obscure peri-articular structures or may mimic disease. Typical sites of leakage are the biceps tendon sheath and underneath the subscapularis muscle in the shoulder (Fig. 8), into the pre-humeral or retro-humeral fat pad in

Table 2 Typical injection volumes, in millilitress ( $L A$ local anaesthetics, Iodine iodine-containing contrast medium, $G d$ gadoliniumcontaining contrast medium)

\begin{tabular}{lllll}
\hline At Balgrist, Zurich & & & & \multicolumn{2}{c}{ Review $^{\text {a }}$} \\
\cline { 1 - 2 } Joint & LA & Iodine & Gd & Gd \\
\hline Glenohumeral & 1 & 1 & 10 & $10-20$ \\
Elbow & 1 & 1 & 5 & - \\
Wrist intercarpal & 0.5 & 1 & 2 & 4 \\
Wrist distal radio-ulnar & Drop & Drop & 1 & - \\
Hip & 1 & 1 & 10 & $10-20$ \\
Knee & 1 & 10 & 20 & $25-50$ \\
Ankle & 1 & 1 & 4 & $12-20$ \\
\hline
\end{tabular}

${ }^{\mathrm{a}}[13]$

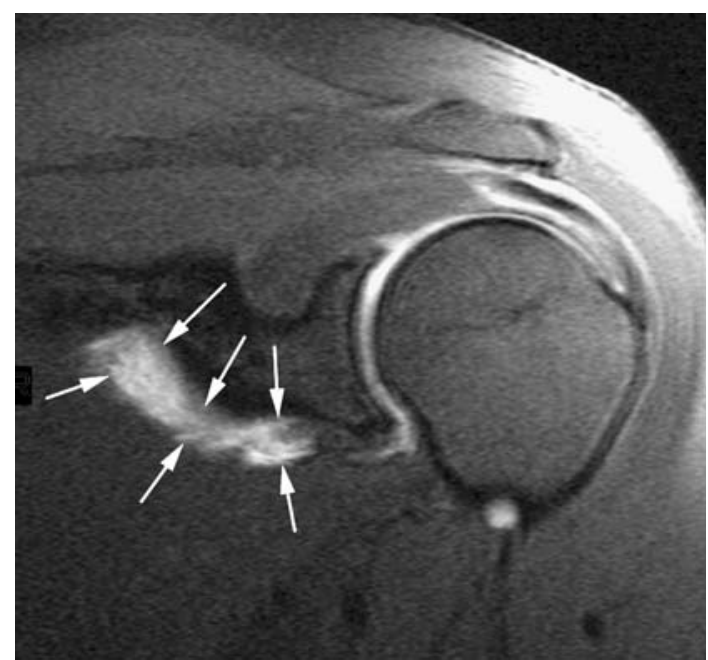

Fig. 8 Contrast leakage. Angled, coronal, fat-suppressed, T1-weighted, spin-echo image after injection of $10 \mathrm{ml}$ of contrast medium, shows narrow axillary recess and contrast leakage underneath the subscapularis muscle (arrows) in a patient with a frozen shoulder

the elbow, along the needle track in the wrist, into the pericapsular area of the hip, into Hoffa's fat pad or the prefemoral fat pad above the knee and of pre-tibial fat above the ankle.

Injection volumes are usually adequate when, starting from standard values, clinical findings are taken into consideration. In addition, patients complain when the

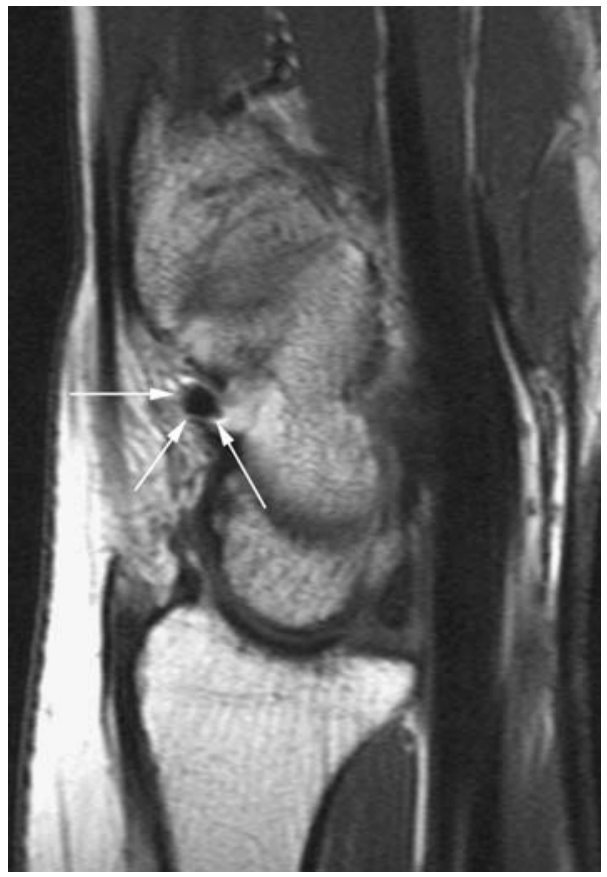

Fig. 9 Susceptibility artefact caused by a small amount of inadvertently injected air. Sagittal T1-weighed image after midcarpal injection (arrows). The air bubble was overlooked when the prolongation tube was filled with contrast agent. Susceptibility artefacts may be more pronounced in gradient echo images 
Fig. 10 Graph comparing gadolinium concentrations (x-axis) and SNR ratios. For sequences typically employed for MR arthrography (such as T1weighted spin-echo) there is quite a broad useful range of concentrations. The concentrations available on the market (2$2.5 \mathrm{mmol} / \mathrm{l})$ are rather in the upper range, which is useful because of the dilution by local anaesthetics, iodine-containing contrast material and pre-existing joint fluid. If the concentration is too low, T2-weighted (fatsuppressed) sequences may be used to salvage an examination. Note complex signal behaviour of the STIR sequence

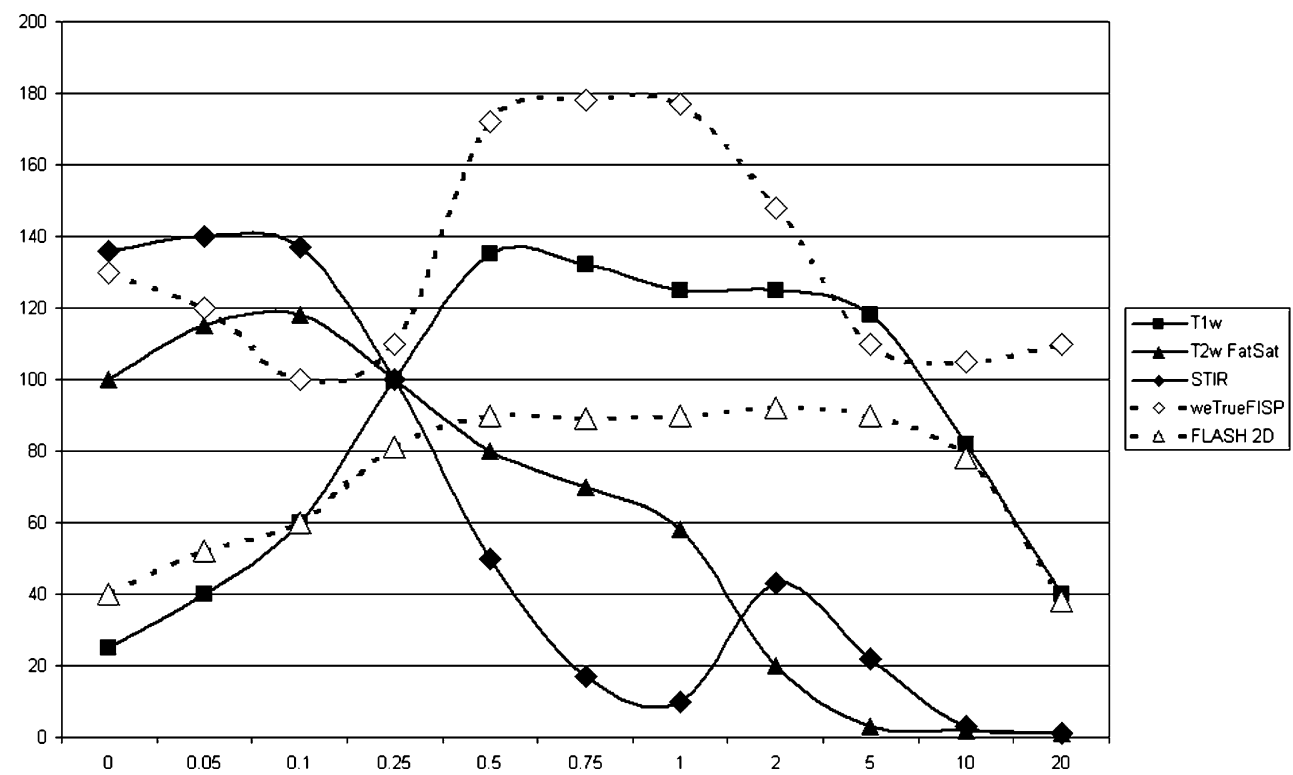

injection volume becomes too large and injection pressure increased.

\section{Injection of room air}

The inadvertent injection of small amounts of room air results in hypointensities that may be difficult to differentiate from intra-articular bodies (Fig. 9). Such air is easily introduced during the changing of syringes. With some of the advanced sequences, such as water-excitation, fatsuppressed, true fast imaging with steady-state precession (trueFISP) and other gradient-echo techniques that allow one to obtain thin slices and good contrast between gadolinium-containing contrast material and articular structures, including cartilage, even small amounts of air may produce quite pronounced susceptibility artefacts. Occa-

sionally, air bubbles may mimic intra-articular bodies, although air tends to be located in the uppermost parts of the joints. Air may also be arranged in small air bubbles along structures such as the biceps tendon within the intertubercular groove [15]. Injection of room air can be avoided quite reliably if a drop of fluid is allowed to fall into the needle hub before the syringe is connected. Some radiologists use three-way-stopcocks to prevent air from entering the joint. In our experience this does not prevent air injection, however, presumably because some residual air may remain within the stopcock.

\section{Infection}

Joint infection in otherwise healthy patients is rarely caused by carefully performed MR arthrography. Based on a

Fig. 11 Contrast and type of sequence. Sagittal, T1-weighted, spin-echo (a) and STIR (b) images of the ankle. Two millimoles per litre of gadopentetate has been injected. On the T1-weighted image, the distended anterior recess is hyperintense, as expected. On the STIR image, the recess is hypointense, presumably because the contrast has been diluted by local anaesthetics and joint fluid
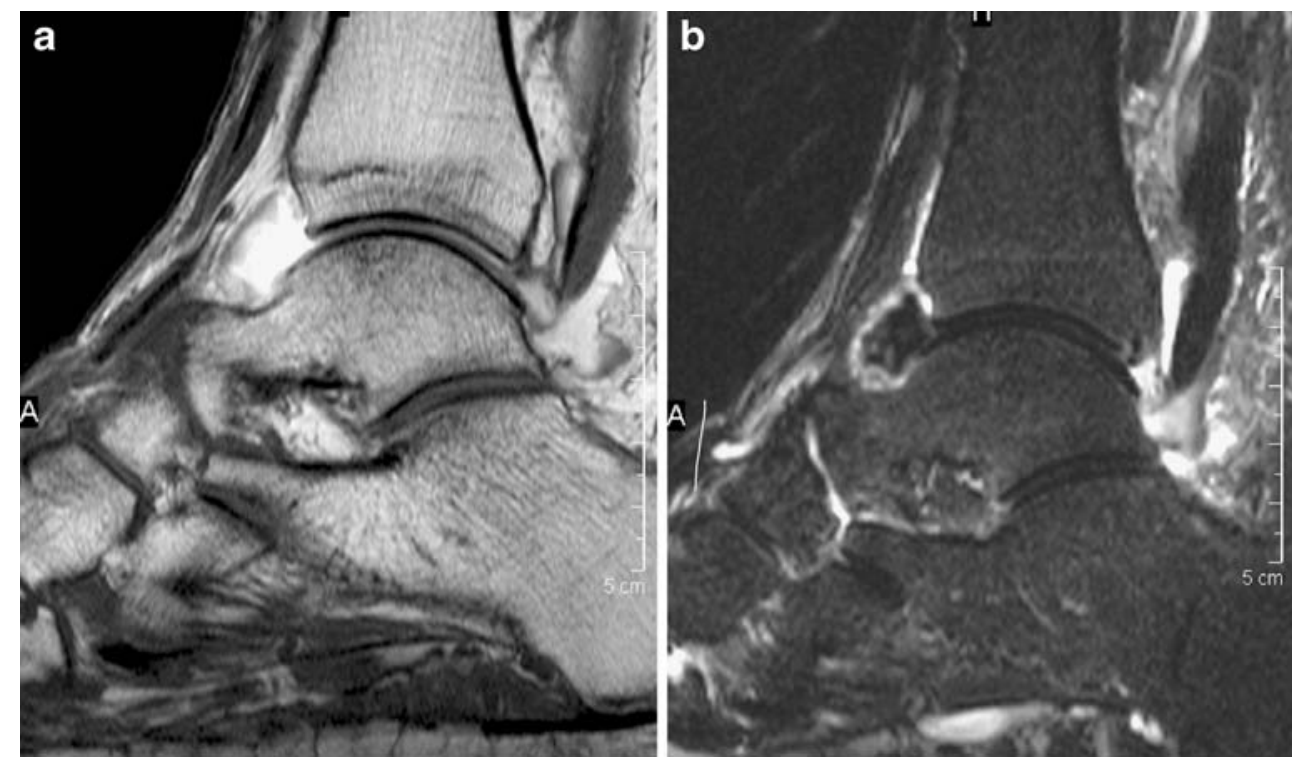

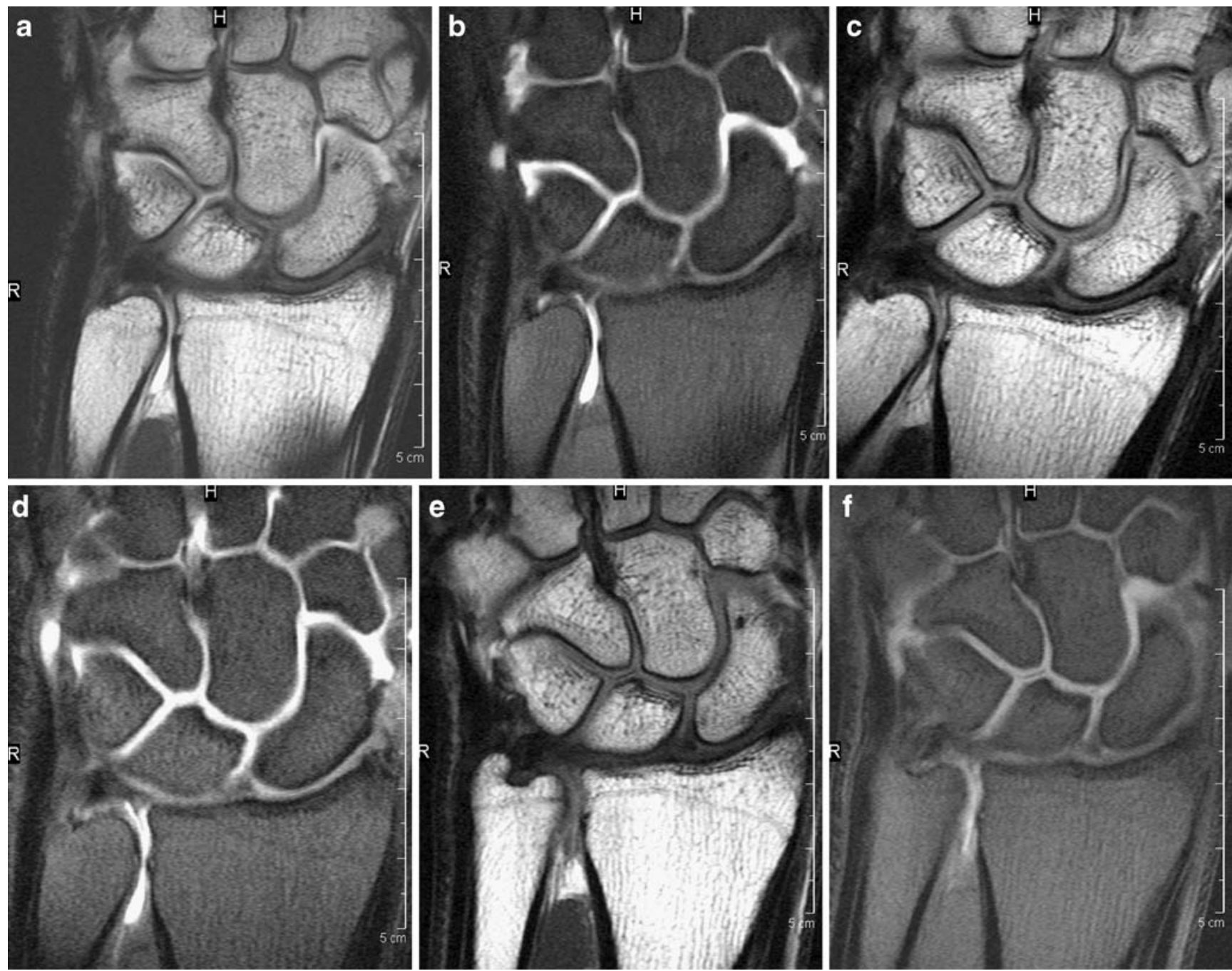

Fig. 12 Temporal behaviour of contrast agent for two different sequence types. Coronal T1-weighted (a,c,e) and T1-weighted fatsuppressed $(\mathbf{b}, \mathbf{d}, \mathbf{f})$ spin-echo images obtained within a few minutes $(\mathbf{a}, \mathbf{b}), 1 \mathrm{~h}(\mathbf{c}, \mathbf{d})$ and $3 \mathrm{~h}(\mathbf{e}, \mathbf{f})$ after contrast injection $(2.5 \mathrm{mmol} / \mathrm{l})$ into the distal radio-ulnar joint. Shortly after injection, both sequences demonstrate hyperintensity within the joint space. After $1 \mathrm{~h}$, the joint

contents are nearly isointense with surrounding fatty tissue on the T1-weighed sequence, with more pronounced contrast on the fatsuppressed image. After $3 \mathrm{~h}$, only a very small amount of slightly hyperintense joint fluid is found on the fat-suppressed image, but not in the standard sequence

questionnaire, Newberg et al. [16] found only three cases of infection in 126,000 arthrographic procedures. In a large review of arthrography, Hugo et al. [17] did not find a single infection associated with MR arthrography $(n=\sim 13,300)$. For the general population (not a cohort of patients who had undergone MR arthrography), the risk of developing septic arthritis is increased in persons above the age of 80 years [odds ratio $(\mathrm{OR}=3.5)$ ], diabetes mellitus $(\mathrm{OR}=3.3)$, rheumatoid arthritis $(\mathrm{OR}=4.0)$, hip and/or knee prosthesis $(\mathrm{OR}=15$, $95 \%)$, joint surgery $(\mathrm{OR}=5.1)$, and skin infection $(\mathrm{OR}=27.2)$ [18]. These numbers indicate that radiologists should be especially careful in these diagnoses when performing MR arthrography.

For articular injections, thorough preparation, masks for the patient, radiologist and other persons staying in the room (including technicians and parents), sterile gloves and the following of general rules of sterility are required.

\section{Contrast media concentrations}

In an early cadaveric study [19], the lowest concentration of gadopentetate achieving diagnostic image quality was $0.5 \mathrm{mmol} / 1$ for standard T1-weighted spin-echo images. Based on our own, unpublished data, the highest signalto-noise ratio is reached between $0.5 \mathrm{mmol} / \mathrm{l}$ and $2 \mathrm{mmol} / \mathrm{L}$ for T1-weighted spin-echo images and $0.25 \mathrm{mmol} / \mathrm{l}$ and $2 \mathrm{mmol} / \mathrm{l}$ for a T1-weighted 2D fast low-angle shot (FLASH) sequence. For a T2-weighted fat-suppressed sequence, the signal-to-noise (SNR) ratio is best for saline 
and low gadolinium concentrations. With increasing gadolinium concentrations the SNR ratio decreases and approaches zero at $2 \mathrm{mmol} / \mathrm{l}$. The STIR sequence has an interesting behaviour. Signal is high when the contrast concentration is very low. At $0.75-1 \mathrm{mmol} / \mathrm{l}$ the signal intensity approaches zero, slightly increases at $2 \mathrm{mmol} / \mathrm{l}$ and again approaches zero above $5 \mathrm{mmol} / \mathrm{l}$ (Figs. 10 and 11).

Laboratory data cannot necessarily be transferred to clinical imaging, because there may be variable amounts of pre-existing joint fluid diluting the contrast medium. Schweitzer et al. [20] found variable amounts of fluid in the glenohumeral joint, which was larger in older patients and in the presence of osteophytes and rotator cuff tears. In the ankle, fluid is very common. In a study involving normal and abnormal ankles, most patients had fluid in the ankle (77\%) and subtalar joints (72\%) [21].

For clinical imaging, concentrations of $2 \mathrm{mmol} / \mathrm{l}$ or $2.5 \mathrm{mmol} / \mathrm{l}$ are commonly and successfully employed for MR arthrography [14, 22]. This corresponds to a dilution of $1: 250$ and $1: 200$, respectively, of the $0.5 \mathrm{~mol} / 1(500 \mathrm{mmol} / \mathrm{l})$ solutions of gadolinium-containing contrast media typically used for intravenous injection. Four millimoles per litre and $5 \mathrm{mmol} / 1$ concentrations or higher $[9,23]$ have been used as well. In a study by Binkert et al. [9] the $4 \mathrm{mmol} / \mathrm{l}$ concentration performed minimally better than a $2 \mathrm{mmol} / \mathrm{l}$ solution in imaging of the glenohumeral joint. Several contrast media manufacturers are marketing ready-to-inject syringes (gadopentetate $2 \mathrm{mmol} / \mathrm{l}$, Magnevist, Schering, Berlin, Germany, Gd-DOTA 2.5 mmol/l, Artirem, Guerbet,
Aulnay-sous-Bois, France) or vials (Gd-DOTA $2.5 \mathrm{mmol} / \mathrm{l}$, Artirem, Guerbet, (Suisse), Zurich, Switzerland).

Rarely, due to errors in handling of contrast material, concentrations intended for intravenous use may be injected into the joint. This results in very low signal, possibly relating to susceptibility artefacts. After a waiting period of several hours, concentrations typically are diluted to diagnostic levels, allowing the performance of a diagnostic MR examination.

\section{Problems relating to MR imaging}

Interval between injection of contrast agent and MR imaging

We typically start MR imaging no later than 15 min after injection. Others use a maximum of 30 min delay [22]. It is important to keep in mind that, depending on the imaging protocols, the last sequence may easily be obtained 30 45 min after the beginning of the examination. Our own data (not published) indicate that imaging should be terminated within $90 \mathrm{~min}$ after injection of the shoulder and hip and within $45 \mathrm{~min}$ after wrist injections (Fig. 12).

A "just-in-time" MR arthrogram requires sufficient fluoroscopy capacity in combination with flexible scheduling of technicians and radiologists. If this is not possible, alternative injection techniques may be employed (such as

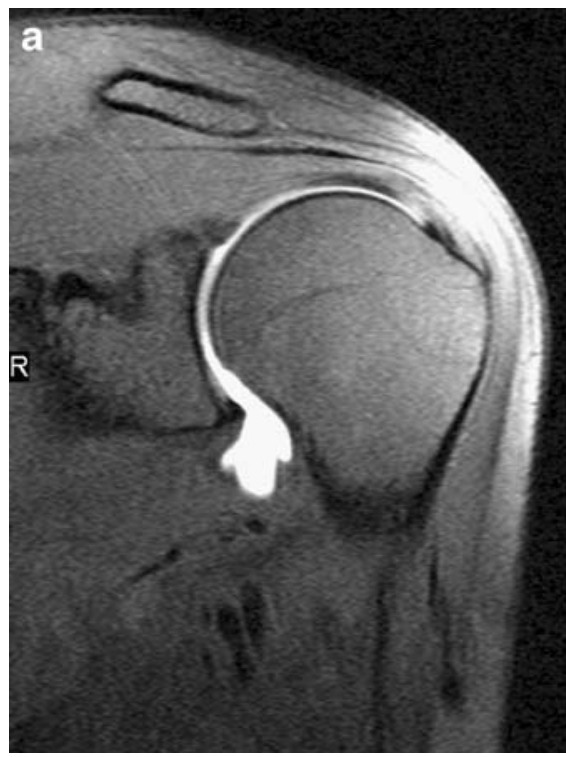

Fig. 13 Potentially missed abnormalities due to examination technique dedicated to MR arthrography. Angled, coronal, fat-suppressed, T1-weighted (a) and T2-weighted (b) images after injection of intraarticular contrast agent, and axial, fat-suppressed, T1-weighted image with larger field-of-view, obtained after intravenous injection of contrast agent (c). The fat-suppressed, T1-weighted, MR arthrographic
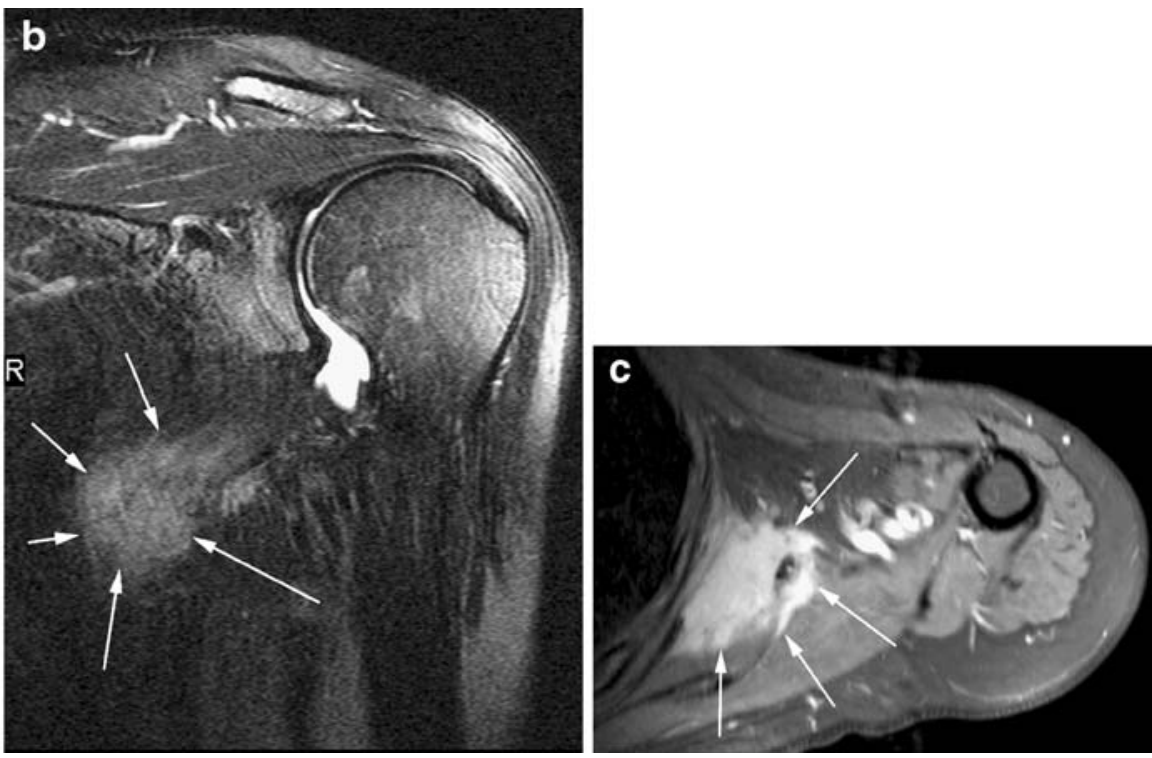

image does not demonstrate the tumour seen on the non-magnified, fat-suppressed, T2-weighted image (arrows in b) and the fat-suppressed T1-weighted image obtained after intravenous injection (arrows in c). This was a desmoid tumour located between the subscapularis and teres major on one hand and the thoracic wall on the other hand 
ultrasound or even MR guidance or a blind injection). Another solution is to increase the concentration of the injected contrast agent. Kopka et al. [24]. found that imaging was possible within $1 \mathrm{~h}$ after injection of $10 \mathrm{mmol} / 1$ of contrast material and $1.5-3 \mathrm{~h}$ after injection of $45 \mathrm{mmol} / \mathrm{l}$ of contrast material. Wagner et al. [25] stated that the potential time intervals varied according to the injected joint. After injection of $2-3 \mathrm{mmol} / \mathrm{l}$ contrast material, and using T1-weighted images with fat suppression, image acquisition was possible $1 \mathrm{~h}$ after injection for the shoulder, after $2 \mathrm{~h}$ for the hip and after $3.5 \mathrm{~h}$ for the knee.

If unexpected delays between injection and MR imaging occur, for instance due to technical problems, the examination may still be performed with STIR or frequencyselective, T2-weighted, spin-echo images which produce good contrast between the remaining intra-articular fluid and surrounding structures.

Missed findings outside the joint space

Sequences suitable for MR arthrography may not demonstrate findings outside the joint space and include T1weighted, fat-suppressed, spin-echo sequences and a number of gradient-echo sequences. If the examination is limited to this type of sequence, soft tissue tumors (Fig. 13), fatty muscle degeneration and bone marrow abnormalities may be missed. Such problems can be avoided by the addition of at least one fat-suppressed, proton-density or T2-weighted sequence or a STIR sequence. In suspected fatty degeneration of muscles, T1-weighted spin-echo images without fat suppression should be employed.

\section{Conclusion}

There are a number of errors that may be committed when MR arthrography is being performed, including the overlooking of contraindications, errors in injection technique and errors relating to contrast dilution, injection volume, local anaesthetics and pulse sequences. However, the potential problems with MR arthrography can usually be solved rather easily.

\section{References}

1. Steinbach LS, Palmer WE, Schweitzer ME. Special focus session. MR arthrography. Radiographics 2002; 22: 1223-1246.

2. Grainger AJ, Elliott JM, Campbell RS, Tirman PF, Steinbach LS, Genant HK. Direct MR arthrography: a review of current use. Clin Radiol 2000; 55: 163-176.

3. Elentuck D, Palmer WE. Direct magnetic resonance arthrography. Eur Radiol 2004; 14: 1956-1967.
4. Dunn AS, Turpie AG. Perioperative management of patients receiving oral anticoagulants: a systematic review. Arch Intern Med 2003; 163: 901-908.

5. Thumboo J, O'Duffy JD. A prospective study of the safety of joint and soft tissue aspirations and injections in patients taking warfarin sodium. Arthritis Rheum 1998; 41: 736-739.

6. Sparling M, Malleson P, Wood B, Petty R. Radiographic followup of joints injected with triamcinolone hexacetonide for the management of childhood arthritis. Arthritis Rheum 1990; 33: 821-826.

7. McCarthy JC, Lee JA. Arthroscopic intervention in early hip disease. Clin Orthop Relat Res 2004; 429: 157-162.

8. Hasselbacher P, Schumacher HR. Synovial fluid eosinophilia following arthrography. J Rheumatol 1978; 5: 173-176.

9. Binkert CA, Zanetti M, Gerber C, Hodler J. MR arthrography of the glenohumeral joint: two concentrations of gadoteridol versus Ringer solution as the intraarticular contrast material. Radiology 2001; 220: 219-224.

10. Zanetti M, Hodler J. Contrast media in MR arthrography of the glenohumeral joint: intra-articular gadopentetate vs saline: preliminary results. Eur Radiol 1997; 7: 498-502.

11. Kuo PH, Kanal E, Abu-Alfa AK, Cowper SE. Gadolinium-based MR contrast agents and nephrogenic systemic fibrosis. Radiology 2007; 242: 647-649.

12. Duc SR, Hodler J, Schmid MR, et al. Prospective evaluation of two different injection techniques for MR arthrography of the hip. Eur Radiol 2006; 16: 473-478.

13. Farmer KD, Hughes PM. MR arthrography of the shoulder: fluoroscopically guided technique using a posterior approach. AJR Am J Roentgenol 2002; 178: 433-434.

14. Schulte-Altedorneburg G, Gebhard M, Wohlgemuth WA, et al. MR arthrography: pharmacology, efficacy and safety in clinical trials. Skeletal Radiol 2003; 32: 1-12.

15. Guckel C, Nidecker A. The rope ladder: an uncommon artifact and potential pitfall in MR arthrography of the shoulder. AJR Am J Roentgenol 1997; 168: 947-950.

16. Newberg AH, Munn CS, Robbins AH. Complications of arthrography. Radiology 1985; 155: 605-606.

17. Hugo PC 3rd, Newberg AH, Newman JS, Wetzner SM. Complications of arthrography. Semin Musculoskelet Radiol 1998; 2: 345-348.

18. Kaandorp CJ, Van Schaardenburg D, Krijnen P, Habbema JD, van de Laar MA. Risk factors for septic arthritis in patients with joint disease. A prospective study. Arthritis Rheum 1995; 38: 1819-1825.

19. Hajek PC, Sartoris DJ, Neumann CH, Resnick D. Potential contrast agents for MR arthrography: in vitro evaluation and practical observations. AJR Am J Roentgenol 1987; 149: 97-104.

20. Schweitzer ME, Magbalon MJ, Fenlin JM, Frieman BG, Ehrlich $\mathrm{S}$, Epstein RE. Effusion criteria and clinical importance of glenohumeral joint fluid: MR imaging evaluation. Radiology 1995; 194: 821-824.

21. Schweitzer ME, van Leersum M, Ehrlich SS, Wapner K. Fluid in normal and abnormal ankle joints: amount and distribution as seen on MR images. AJR Am J Roentgenol 1994; 162: 111-114.

22. Brenner ML, Morrison WB, Carrino JA, et al. Direct MR arthrography of the shoulder: is exercise prior to imaging beneficial or detrimental? Radiology 2000; 215: 491-496.

23. Smith DK, Chopp TM, Aufdemorte TB, Witkowski EG, Jones RC. Sublabral recess of the superior glenoid labrum: study of cadavers with conventional nonenhanced MR imaging, MR arthrography, anatomic dissection, and limited histologic examination. Radiology 1996; 201: 251-256.

24. Kopka L, Funke M, Fischer U, Keating D, Oestmann J, Grabbe E. MR arthrography of the shoulder with gadopentetate dimeglumine: influence of concentration, iodinated contrast material, and time on signal intensity. AJR Am J Roentgenol 1994; 163: 621-623.

25. Wagner SC, Schweitzer ME, Weishaupt D. Temporal behavior of intraarticular gadolinium. J Comput Assist Tomogr 2001; 25: 661-670. 